\title{
Functional significance of B7-H1 expressed by human uveal melanoma cells
}

\author{
RENBING JIA $^{1 *}$, ZHIJUN JIAO ${ }^{2 *}$, XIAOFANG XU ${ }^{1}$, JING WANG ${ }^{1}$, YIXIONG ZHOU ${ }^{1}$, \\ XIN SONG ${ }^{1}$, SHENGFANG GE ${ }^{1}$ and XIANQUN FAN ${ }^{1}$ \\ ${ }^{1}$ Department of Ophthalmology, Ninth People's Hospital, Shanghai Jiaotong University School of Medicine, Shanghai 200011; \\ ${ }^{2}$ Department of Laboratory Medicine, Affiliated Hospital of Jiangsu University, Zhenjiang, Jiangsu 212001, P.R. China
}

Received August 18, 2010; Accepted November 1, 2010

DOI: $10.3892 / \mathrm{mmr} .2010 .397$

\begin{abstract}
B7-H1, a recently described B7 family member, has been reported to negatively regulate $\mathrm{T}$-cell function in most cancer cells. In this study, we sought to investigate B7-H1 expression in four uveal melanoma (UM) cells (OCM1, SP6.5, OM431 and VUP) to determine the functional significance of B7-H1 expression in T-cell immune response. Using flow cytometry (FCM), we demonstrated that SP6.5 cells had high B7-H1 protein expression, while the other three UM cell lines had none. However, all four UM cell lines expressed B7-H1 mRNA, as confirmed by reverse transcription-polymerase chain reaction. In co-culture experiments using B7-H1expressing UM cells with T-cells, FCM to determine CD69 expression in T-cells revealed that SP6.5 cell-related B7-H1 inhibited T-cell activation. This effect was eliminated by B7-H1-targeted RNA interference. An Annexin V/PI double staining assay further showed that B7-H1 expressed by SP6.5 cells did not increase the apoptosis of T-cells, though it was found in a variety of other solid tumors. In conclusion, all the UM cell lines constitutively expressed B7-H1 mRNA, while B7-H1 protein was expressed at different levels. UM-related B7-H1 expression negatively regulated T-cell immune response through the inhibition of T-cell activation, and not through the promotion of T-cell apoptosis. This provides new insight into anti-tumor immunity against B7-H1-expressing UM cells.
\end{abstract}

\section{Introduction}

Uveal melanoma (UM) is the most common primary intraocular tumor in adults (1), and has a strong propensity to spread

Correspondence to: Dr Xianqun Fan and Dr Shengfang Ge, Department of Ophthalmology, Ninth People's Hospital, Shanghai Jiaotong University School of Medicine, 639 Zhi Zao Ju Road, Shanghai 200011, P.R. China

E-mail: fanxq@sh163.net; geshengfang@hotmail.com

*Contributed equally

Key words: uveal melanoma, B7-H1, tumor immunity to the liver (2). It has been shown that hepatic micrometastases of UM occur a few years before the diagnosis of the primary intraocular malignancy $(3,4)$. Irrespective of the success of treatment of the primary tumor, UM therapy remains problematic due to the high rate of metastatic dissemination. In cases of metastatic disease, patient prognosis is poor; the disease is usually fatal within 1 year.

B7-H1 is a recently described B7 family member that has a profound effect on the regulation of $\mathrm{T}$-cell response. B7-H1 mRNA is broadly distributed in normal tissues and organs (5-8), while the protein expression of B7-H1 is found in macrophage-like cells. However, B7-H1 protein is aberrantly expressed by tumor cell lines and tumor tissues, including lung, ovarian and breast carcinomas $(9,10)$. The role of B7-H1 in the regulation of T-cell response in inflammation and autoimmunity appears to be complex and remains under debate. However, accumulating evidence indicates that the interaction of B7-H1 with programmed death-1 (PD-1) may induce a negative regulatory signal on tumor immunity by inhibiting T-cell activation or/and inducing T-cell apoptosis (11). More recently, it was reported that B7-H1 expressed by several UM cell lines regulated T-cell function by suppressing interleukin-2 production (12).

In this study, we examined four UM cell lines and found that all four expressed B7-H1 mRNA, while only one expressed B7-H1 protein. Co-culture experiments demonstrated that the high B7-H1 expression in SP6.5 cells reduced T-cell activation, while UM-related B7-H1 expression had no effect on T-cell apoptosis. Thus, this study provides further insight into the functional significance of B7-H1 in T-cell immune response in UM cells.

\section{Materials and methods}

Cell culture. The human UM cell lines OCM1, SP6.5, OM431 and VUP were kind gifts of John F. Marshall (Tumor Biology Laboratory, Cancer Research UK Clinical Center, John Vane Science Centre, London, UK). It has been established that UMs are composed of spindle and epithelioid cells, and are histopathologically classified as either spindle-cell or mixed-cell type tumors. All the UM cells used in this study were derived from primary tumors. Among them, the OCM1, OM431 and VUP cell lines were predominantly composed of 
epithelioid cells, whereas the SP6.5 cell line was a spindleepithelioid mixed type culture from a primary tumor comprising essentially spindle-like cells. The UM cell lines were cultured in DMEM supplemented with $10 \%$ FCS, $2 \mathrm{mM}$ L-glutamine, $100 \mathrm{U} / \mathrm{ml}$ streptomycin and $100 \mathrm{mg} / \mathrm{ml}$ penicillin at $37^{\circ} \mathrm{C}$ in a humidified incubator with $5 \% \mathrm{CO}_{2}$.

RNA extraction, cDNA synthesis and reverse transcriptionpolymerase chain reaction (RT-PCR). Total RNAs were isolated using TRIzol reagent (Invitrogen, Carlsbad, CA, USA) according to the manufacturer's instructions. Reagents for the analysis of RT-PCR were purchased from Haojia Inc. (Dalian, China). Total RNA $(1 \mu \mathrm{g})$ was reverse transcribed using Murine Moloney Leukemia Virus reverse transcriptase and oligo-dT primers in $10-\mu 1$ reaction volumes. PCR reactions $(50 \mu \mathrm{l})$ were assembled with final concentrations of $1 \mathrm{X}$ buffer, $1.5 \mathrm{mM} \mathrm{MgCl}, 0.2 \mathrm{mM}$ dNTPs, $0.2 \mu \mathrm{M}$ of each primer and 2.5 units $T a q$ polymerase (Invitrogen). Primers were designed using Primer 5.0 Software (Premier Biosoft International, Palo Alto, CA, USA). The primer sequences were as follows: B7-H1 forward, 5'-TTTCAATGT GACCAGCAC-3'; B7-H1 reverse, 5'-CTTAAACGGAAG ATGAATGT-3'; GAPDH forward, 5'-AACGGATTTGGTCGT ATTG-'3; GAPDH reverse, 5'-GGAAGATGGTGATGGG ATT-'3. PCR cycle parameters were as follows: an initial step at $94^{\circ} \mathrm{C}$ for $2 \mathrm{~min}$, followed by 30 cycles at $94^{\circ} \mathrm{C}$ for $30 \mathrm{sec}$, annealing at $58^{\circ} \mathrm{C}$ for $30 \mathrm{sec}$ and $72^{\circ} \mathrm{C}$ for $30 \mathrm{sec}$, and a final step at $72^{\circ} \mathrm{C}$ for $5 \mathrm{~min}$. The amplified products were analyzed by electrophoresis on $1.5 \%$ agarose gels.

Plasmid construction. The siRNA sequence and siRNA expression vector pSilencer4.1-CMV were purchased from Ambion Inc. (Austin, TX, USA). The sense strand sequences were: B7-H1, 5'-UAUGACAAUUGAAUGCAAA-TT; and control, 5'-GAUAGCAAUGACGAAUGCG-TT, which is a scrambled sequence without significant homology to sequences in the human and mouse genomes. SiRNA expressing plasmid p4.1CMV/B7-H1 siRNA targeting human B7-H1 and control plasmid p4.1CMV/CONT siRNA were constructed according to the manufacturer's instructions. Briefly, the cDNA oligonucleotides of B7-H1 siRNA and control siRNA were synthesized by Integrated DNA Technologies (Coralville, IA, USA). Both pairs of oligonucleotides were annealed at $90^{\circ} \mathrm{C}$ for $3 \mathrm{~min}$, cooled to $37^{\circ} \mathrm{C}$, and then incubated for $1 \mathrm{~h}$. The annealed dsDNA oligonucleotides were ligated between the BamHI and HindIII sites on the pSilencer4.1-CMV vector. The inserted sequences were verified by DNA sequencing.

Transfection. SP6.5 cells in the exponential phase of growth were plated in 24 -well plates at $5 \times 10^{4}$ cells/well, grown for $24 \mathrm{~h}$, and then transfected with B7-H1 siRNA expression plasmid and control plasmid using Lipofectamine $2000^{\mathrm{TM}}$ (Invitrogen) according to the manufacturer's instructions. Forty-eight hours after transient transfection, the transfected cells were re-plated with gradient dilution in media containing $600 \mu \mathrm{g} / \mathrm{ml}$ neomycin G418 (Invitrogen). Subsequently, the cells were cultured for 2 weeks, with the selective media replaced when indicated. Individual clones were identified and then enriched under neomycin selection pressure $(400 \mu \mathrm{g} / \mathrm{ml})$ for further experiments.
Real-time RT-PCR. One step SYBR real-time PCR of B7-H1 mRNA was performed according to the manufacturer's instructions (Takara, Tokyo, Japan). Briefly, $2 \mu \mathrm{g}$ of RNA per sample was reverse transcribed into cDNA using random primers. The reverse transcription reaction consisted of $42^{\circ} \mathrm{C}$ for $15 \mathrm{~min}$ and $95^{\circ} \mathrm{C}$ for $2 \mathrm{~min}$. Specific PCR primers for B7-H1 were: forward, 5'-AGAACTACCTCTGGCACAT-'3; reverse, 5'-ATCCATCATTCTCCCTTT-'3. GAPDH primers were: forward, 5'-AACGGATTTGGTCGTATTG-'3; reverse, 5'-GGAAGATGGTGATGGGATT-'3. The PCR reaction consisted of $30 \mathrm{~min}$ at $50^{\circ} \mathrm{C}$ for cDNA synthesis, followed by amplification for 45 cycles at $95^{\circ} \mathrm{C}$ for $15 \mathrm{sec}, 55^{\circ} \mathrm{C}$ for $30 \mathrm{sec}$ and $72^{\circ} \mathrm{C}$ for $30 \mathrm{sec}$. Reactions were performed and data analyzed using the LightCycler ${ }^{\circledR}$ system (Roche Diagnostics Inc., Switzerland). Results were analyzed by comparing $2^{-\Delta \Delta \mathrm{Ct}}$ values of B7-H1 mRNA in p4.1CMV/B7-H1 siRNAtransfected cells and control.

Purified lymphocyte populations and co-culture. Peripheral blood mononuclear cells (PBMCs) were isolated from the peripheral blood of normal healthy volunteers by Ficoll density gradient centrifugation. T-cells $\left(\mathrm{CD}^{+}\right.$and $\left.\mathrm{CD}^{+}\right)$were purified using microbeads (Miltenyi Biotec, Bergisch Gladbach, Germany) according to the provider's instructions. Co-culture experiments were carried out as follows: in brief, purified T-cells were collected by centrifugation and resuspended at a concentration of $2 \times 10^{6}$ cells $/ \mathrm{ml}$ in complete DMEM with the addition of $5 \mathrm{mg} / \mathrm{ml}$ super-antigen Staphylococcus enterotoxin B (SEB) to stimulate T-cell activation, then added to $100-\mathrm{mm}$ dishes containing UM cells at a $2: 1$ tumor/T-cell ratio. Following a 48 -h incubation period, the non-adherent T-cells were removed from the UM cell monolayer and assessed for the expression of T-cell activation marker CD69 by flow cytometry (FCM).

Flow cytometry. Phycoerythrin (PE)-labeled monoclonal antibodies (mAbs) of B7-H1 and T-cell activation marker CD69 were purchased from BD Bioscience (San Jose, CA, USA). To assess B7-H1 and CD69 expression, FCM was performed using standard methods. Briefly, the cells were centrifuged, washed, resuspended in phosphate buffered saline (PBS) and stained at $4^{\circ} \mathrm{C}$ for $30 \mathrm{~min}$ with PE-labeled anti-B7-H1 mAb or PE-labeled anti-CD69 mAb. Then, the cells were washed again and analyzed using a FACSCalibur flow cytometer with CellQuest software (both Beckton Dickinson). The data are presented as the percentage of surface molecule-expressed cells. In each case, appropriately labeled isotype control antibody was used.

Annexin V/PI staining. After a 24-h co-culture, T-cells were collected for Annexin V/PI staining according to the procedures described by BD Bioscience. In brief, T-cells were washed twice with cold PBS and then resuspended in $1 \mathrm{X}$ binding buffer at a concentration of $1 \times 10^{6}$ cells $/ \mathrm{ml}$. Subsequently, $100 \mu \mathrm{l}$ of the solution was transferred into a 5-ml culture tube, and $5 \mu \mathrm{l}$ Annexin V and $2 \mu \mathrm{l}$ PI was added. The cells were gently mixed then incubated for $15 \mathrm{~min}$ at room tempearture in the dark. Finally, $400 \mu \mathrm{l}$ of $1 \mathrm{X}$ binding buffer was added to each tube, followed within $30 \mathrm{~min}$ by FCM analysis. 
A

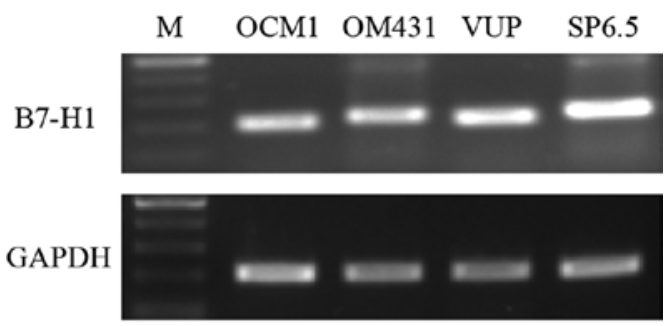

B
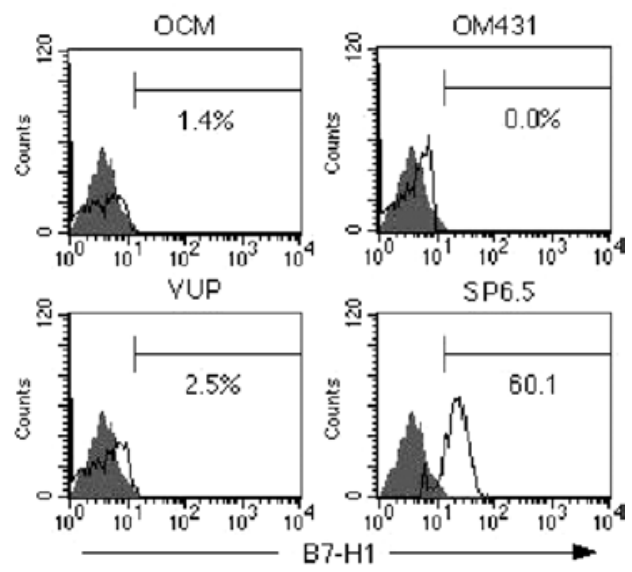

Figure 1. B7-H1 mRNA and protein expression in human UM cells. (A) B7-H1 mRNA expression in the four tested UM cell lines determined by RT-PCR. (B) B7-H1 protein expression at the cell surface analyzed by flow cytometry. Representative results are shown.

\section{Results}

B7-H1 expression in UM cell lines. The exact nature of UM B7-H1 expression is unclear. Thus, four UM cell lines (OCM1, VUP, SP6.5 and OM431) were first tested for B7-H1 expression at both the mRNA and protein levels. As determined by FCM, all of the UM cell lines constitutively expressed moderate to high levels of B7-H1 mRNA (Fig. 1A), while constitutive B7-H1 molecule expression was only observed in the SP6.5 UM cell line (Fig. 1B). This suggests that UM B7-H1 expression is controlled by posttranscriptional mechanisms.

Inhibition of B7-H1 expression by plasmid-based RNAi. To determine the blocking effect of vector-based RNAi on B7-H1 expression, B7-H1 siRNA-expressing plasmid p4.1CMV/B7-H1 siRNA and control plasmid p4.1CMV/ CONT siRNA, which encodes a scrambled sequence without significant homology to sequences in the human and mouse genomes, were constructed and stably transfected into B7-H1-expressing SP6.5 cells. Compared to p4.1CMV/ B7-H1 siRNA-transfected cells (set as 100\%), B7-H1 mRNA levels were $714.33 \%$ in $\mathrm{p} 4.1 \mathrm{CMV} / \mathrm{CONT}$ siRNA-transfected cells and $588.29 \%$ in untransfected cells. Representative results of three independent FCM experiments performed to detect B7-H1 protein expression are shown in Fig. 2A. The results show a significant reduction in B7-H1 protein levels in transfectants $(8.44 \pm 3.56 \%)$ compared to the controls $(63.37 \pm 14.22 \%$ and $64.20 \pm 8.59 \%)$ (Fig. 2B), suggesting that vector-based RNAi, rather than anti-B7-H1 mAb, may be reliably used to block B7-H1.

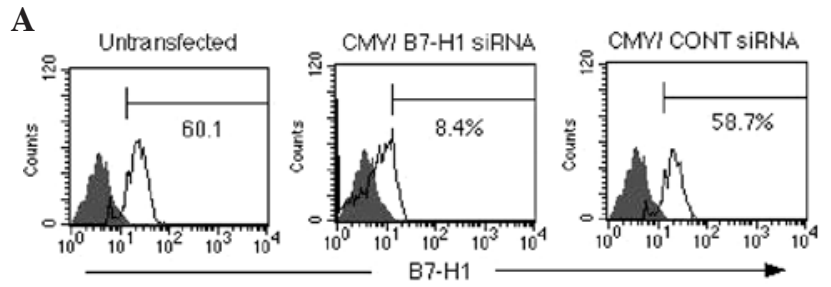

B

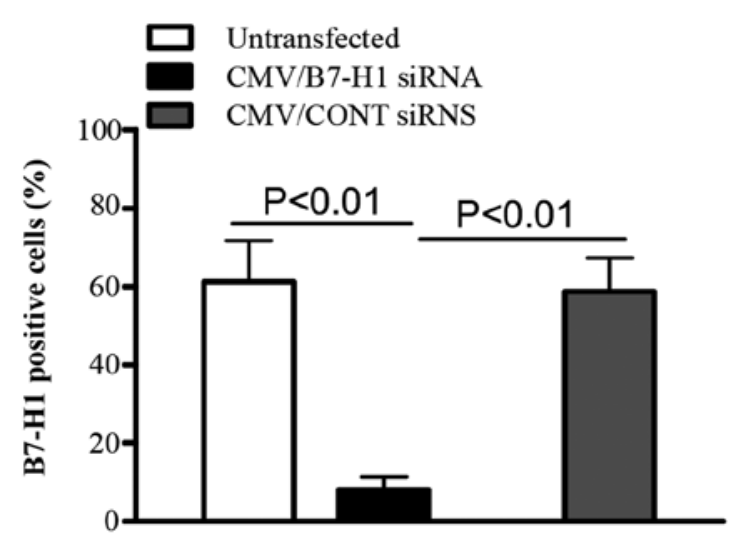

Figure 2. Effect of plasmid-based RNAi on SP6.5 cell B7-H1 expression. SP6.5 cells were stably transfected with B7-H1 siRNA construct and control construct. B7-H1 mRNA was quantified by real-time PCR. The relative level of B7-H1 mRNA in p4.1CMV/B7-H1 siRNA-transfected cells compared to the control was calculated using the $2^{-\Delta \Delta \mathrm{Ct}}$ method. Compared to p4.1CMV/B7-H1 siRNA-transfected cells (set as 100\%), the relative B7-H1 mRNA levels were $714.33 \%$ in p4.1CMV/CONT siRNA-transfected cells and $588.29 \%$ in mock transfected cells. (A) B7-H1 protein levels on the surface of SP6.5 cells measured by flow cytometry. Representative results from three independent experiments are shown. (B) Data are presented as the means \pm SD. Significant differences were found between the p4.1CMV/ B7-H1 siRNA-transfected cells and the control.

Inhibition of T-cell activation by B7-H1. To investigate the effect of endogenous B7-H1 expression on UM cells, co-culture experiments were performed using SEB-stimulated T-cells and SP6.5 cells transfected with p4.1 CMV/B7-H1 siRNA. The level of activated T-cells was determined by FCM for CD69. Representative results of three experiments are shown in Fig. 3A. T-cell activation was found to be significantly increased in p4.1CMV/B7-H1 siRNA-transfected SP6.5 cells compared to the controls (Fig. 3B), indicating that B7-H1 may be an inhibitor of T-cell activation in B7-H1-expressing UM.

No induction of T-cell apoptosis by B7-H1. It has been reported that B7-H1 may exert an anti-tumor effect through the induction of T-cell apoptosis. We therefore sought to determine whether UM-related B7-H1 promotes T-cell apoptosis. Representative results of Annexin V/PI staining performed three times are shown in Fig. 4A. Notably, no significant increase in T-cell apoptosis was found after co-culture of T-cells with B7-H1expressing SP6.5 cells (Fig. 4B), suggesting that UM-related B7-H1 does not influence T-cell behavior by inducing apoptosis.

\section{Discussion}

B7-H1 has been found to be expressed in a wide range of tumors, including extraocular melanoma (13). Recently, UM B7-H1 was reported to also be expressed in several UM cell 
A

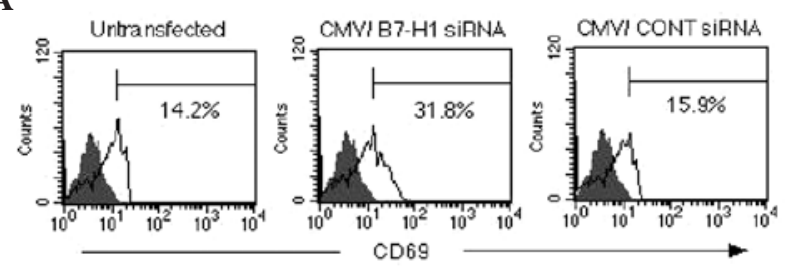

B

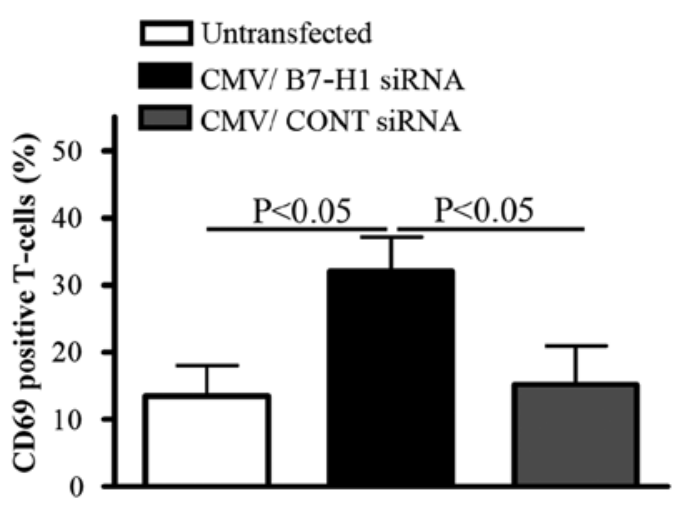

Figure 3. Effect of B7-H1 expression on T-cell activation. SP6.5 cells were stably transfected with B7-H1 siRNA construct or control construct, then co-culture experiments using transfected SP6.5 and control SP6.5 cells with SEB-stimulated T-cells were performed. Non-adherent T-cells were collected by centrifugation $24 \mathrm{~h}$ after co-culture. (A) Levels of T-cell activation marker CD69 measured by flow cytometry. Representative results from three independent experiments are shown. (B) Data are presented as the means \pm SD. Significant differences were found $(\mathrm{p}<0.01)$.

lines (12). In this study, we found B7-H1 mRNA expression in two UM cell lines, OCM1 and OM431, similar to that found previously, and also observed B7-H1 mRNA to be constitutively expressed in two additional UM cell lines, SP6.5 and VUP. B7-H1 protein was expressed at high levels in only one of the cell lines, SP6.5. Of note, UM-associated B7-H1 was found to lead to the inhibition of T-cell activation, even without IFN-stimulation. Furthermore, it appears that UM-related B7-H1 expression has no functional effect on T-cell apoptosis.

B7-H1 is a type I transmembrane protein with an amino acid identity score of $20 \%$ for B7.1 and $15 \%$ for B7.2 (14). B7-H1 interacts with PD-1 and with an as yet unidentified non-PD-1 receptor located on T-cells (15). To date, tumorexpressed B7-H1 has nearly always been shown to exert a potent negative regulatory function on T-cell response, which contributes to tumor progression $(11,12,16-18)$. However, B7-H1 also plays protective roles in pathological conditions, such as inflammation (19). The mechanisms responsible for the inhibitory effect of B7-H1 on T-cell response are still not fully understood, though two possible mechanisms, inhibition of T-cell activation and promotion of T-cell apoptosis, have been proposed based on independent experimental systems. Glioma-related B7-H1 has been identified as a strong inhibitor of T-cell activation, as reflected by increased cytokine production and increased expression levels of the T-cell activation marker CD69 (10). Another investigation revealed that mouse P815 tumor expression of B7-H1 increases the apoptosis of activated tumor-reactive T-cells and promotes the growth of highly immunogenic B7-H1(+) tumors (9).
A
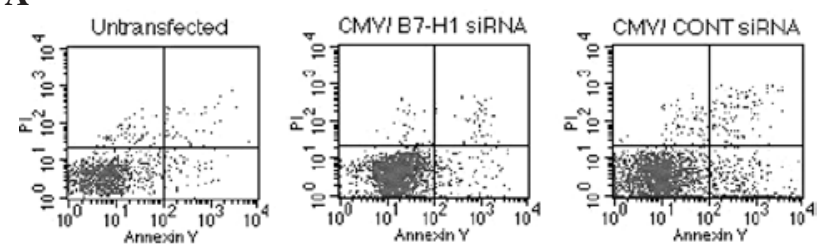

B

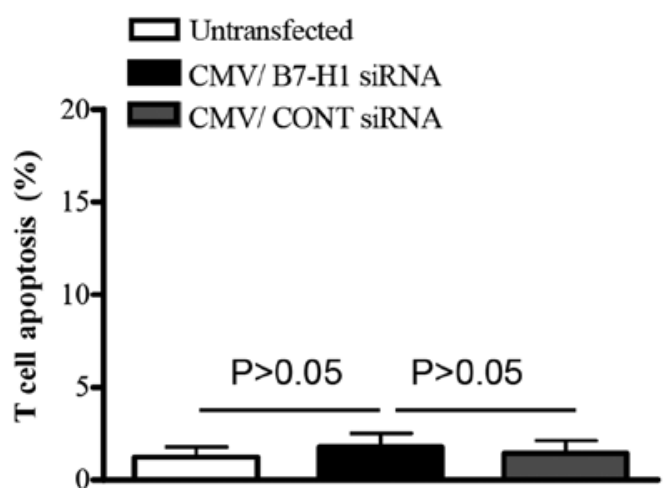

Figure 4. Influence of UM-related B7-H1 expression on T-cell apoptosis. After a 24-h co-culture, T-cells were collected and stained with Annexin V/ PI. (A) Flow cytometry showing apoptotic cells located in the lower right quadrant. Representative results from three independent experiments are shown. (B) Data are presented as the means \pm SD. No significant differences were found $(\mathrm{p}>0.05)$.

Although the expression of B7-H1 mRNA is common in many cells, B7-H1 protein is usually undetectable, suggesting posttranscriptional suppression $(9,20)$. The results of the present study revealed that the levels of B7-H1 protein on the surface of UM cells were not paralleled by B7-H1 mRNA levels. Using semi-quantitative gel stripe assessment of B7-H1 RT-PCR products, no significant difference was found between B7-H1 mRNA levels among the tested UM cells (data not shown). This suggests that tumor-associated B7-H1 expression is also controlled by a posttranscriptional mechanism. However, when and how B7-H1 protein acquires its expression in UM cells remains to be elucidated.

Proinflammatory cytokines such as IFN- $\gamma$ are potent activators for inducing B7-H1 protein expression in T-, B-, endothelial and epithelial cells (21). In vitro experiments have indicated that numerous tumor cell lines up-regulate PD-L1 surface expression upon exposure to IFN- $\gamma(9,10,22)$, and then negatively regulate $\mathrm{T}$-cell function. In this study, we found that natural B7-H1 surface expression in SP6.5 cells inhibits T-cell activation, even without exposure to IFN- $\gamma$. This may partly explain why spindle-like SP6.5 cells are less aggressive than the other three cell lines used. It is worth noting that, in our study, UM-associated B7-H1 did not increase T-cell apoptosis, as determined by FCM using Annexin V and PI double staining. This may be due to the use of a different experimental system and/or the presence of different expression levels of B7-H1 protein.

To the best of our knowledge, all B7-H1-blocking experiments to date have been performed using anti-B7-H1 antibody. However, the lack of cellular cytotoxicity studies remains the major limitation, and must be addressed. Thus, we used plasmid-based RNA interference (RNAi) to block B7-H1 
expression ex vivo. RNAi is a conserved biologic response that results in the sequence-specific silencing of target gene expression. Over the past 5 years, an intensive research effort has facilitated a rapid change in the understanding of RNAi, which has gone from being a relatively obscure biologic phenomenon to a valuable tool used to silence target gene expression and to perform large-scale functional genomic screens (23). To date, several methods have been developed to mediate RNAi; among them, vector-based RNAi is preferable for ex vivo research. The major reason for this is that expression vectors allow continuous production of siRNAs in cells and, therefore, sustained depletion of the protein encoded by the targeted mRNA (24). Once transcribed, a 19- to 21-bp stem-loop structure, termed short-hairpin RNA, is processed by Dicer into a siRNA that directs the down-regulation of target gene expression via elements of the RNAi machinery. In our laboratory, plasmid-based RNAi down-regulated B7-H1 mRNA expression by over $80 \%$, and no obvious side effects were observed. This suggests that plasmid-based RNAi may be used as an alternative method to block B7-H1.

In conclusion, this report characterized B7-H1 mRNA and protein expression in four UM cell lines, including two previously reported cell lines, SP6.5 and VUP. More importantly, our results identified a negative contribution of UM-related B7-H1 to T-cell activation, even under IFN- $\gamma$-free conditions. Additionally, we confirmed that the natural expression of B7-H1 molecules in SP6.5 cells plays no role in T-cell apoptosis. Taken together, this study provides further insight into the functional significance of B7-H1 in UM cells.

\section{Acknowledgements}

This study was supported by the Shanghai Leading Academic Discipline Project (S30205), the National Natural Science Foundation of China (30901654), the Science and Technology Committee of Shanghai (08410702300, 10JC1409100) and the Shanghai Public Health Bureau (2006Y20).

\section{References}

1. Egan KM, Seddon JM, Glynn RJ, Gragoudas ES and Albert DM: Epidemiologic aspects of uveal melanoma. Surv Ophthalmol 32: 239-251, 1988

2. Donoso LA, Berd D, Augsburger JJ, Mastrangelo MJ and Shields JA: Metastatic uveal melanoma: pretherapy serum liver enzyme and liver scan abnormalities. Arch Ophthalmol 103 796-798, 1985.

3. Eskelin S, Pyrhönen S, Summanen P, Hahka-Kemppinen M and Kivelä T: Tumor doubling times in metastatic malignant melanoma of the uvea: tumor progression before and after treatment. Ophthalmology 107: 1443-1449, 2000.

4. Singh AD: Uveal melanoma: implications of tumor doubling time. Ophthalmology 108: 829-830, 2001.

5. Dong H, Zhu G, Tamada K and Chen L: B7-H1, a third member of the B7 family, co-stimulates T-cell proliferation and interleukin-10 secretion. Nat Med 5: 1365-1369, 1999.
6. Wang S, Bajorath J, Flies DB, Dong H, Honjo T and Chen L: Molecular modeling and functional mapping of B7-H1 and B7-DC uncouple costimulatory function from PD-1 interaction. J Exp Med 197: 1083-1091, 2003.

7. Freeman GJ, Long AJ, Iwai Y, et al: Engagement of the PD-1 immunoinhibitory receptor by a novel B7 family member leads to negative regulation of lymphocyte activation. J Exp Med 192: 1027-1034, 2000

8. Tamura H, Dong H, Zhu G, Sica GL, Flies DB, Tamada K and Chen L: B7-H1 costimulation preferentially enhances CD28independent T-helper cell function. Blood 97: 1809-1816, 2001.

9. Dong H, Strome SE, Salomao DR, et al: Tumor-associated B7-H1 promotes T-cell apoptosis: a potential mechanism of immune evasion. Nat Med 8: 793-800, 2002.

10. Wintterle S, Schreiner B, Mitsdoerffer M, et al: Expression of the B7-related molecule B7-H1 by glioma cells: a potential mechanism of immune paralysis. Cancer Res 63: 7462-7467, 2003.

11. Zhang P, Su DM, Liang M and Fu J: Chemopreventive agents induce programmed death-1-ligand 1 (PD-L1) surface expression in breast cancer cells and promote PD-L1-mediated T cell apoptosis. Mol Immunol 45: 1470-1476, 2008.

12. Yang W, Chen PW, Li H, Alizadeh H and Niederkorn JY: PD-L1: PD-1 interaction contributes to the functional suppression of T-cell responses to human uveal melanoma cells in vitro. Invest Ophthalmol Vis Sci 49: 2518-2525, 2008.

13. Blank C, Kuball J, Voelkl S, et al: Blockade of PD-L1 (B7-H1) augments human tumor-specific $\mathrm{T}$ cell responses in vitro. Int J Cancer 119: 317-327, 2006.

14. Wiendl H, Mitsdoerffer M, Schneider D, Chen L, Lochmüller H, Melms A and Weller M: Human muscle cells express a B7-related molecule, B7-H1, with strong negative immune regulatory potential: a novel mechanism of counterbalancing the immune attack in idiopathic inflammatory myopathies. FASEB J 17: 1892-1894, 2003.

15. Magnus T, Schreiner B, Korn T, et al: Microglial expression of the B7 family member B7 homolog 1 confers strong immune inhibition: implications for immune responses and autoimmunity in the CNS. J Neurosci 25: 2537-2546, 2005.

16. Thompson RH, Kuntz SM, Leibovich BC, et al: Tumor B7-H1 is associated with poor prognosis in renal cell carcinoma patients with long-term follow-up. Cancer Res 66: 3381-3385, 2006.

17. Ghebeh H, Mohammed S, Al-Omair A, et al: The B7-H1 (PD-L1) $\mathrm{T}$ lymphocyte-inhibitory molecule is expressed in breast cancer patients with infiltrating ductal carcinoma: correlation with important high-risk prognostic factors. Neoplasia 8: 190-198, 2006.

18. Salih HR, Wintterle S, Krusch M, Kroner A, Huang YH, Chen L and Wiendl $\mathrm{H}$ : The role of leukemia-derived B7-H1 (PD-L1) in tumor-T-cell interactions in humans. Exp Hematol 34: 888-894, 2006.

19. Kanai T, Totsuka T, Uraushihara K, et al: Blockade of B7-H1 suppresses the development of chronic intestinal inflammation. J Immunol 171: 4156-4163, 2003.

20. Yamazaki T, Akiba H, Iwai $\mathrm{H}$, et al: Expression of programmed death 1 ligands by murine T cells and APC. J Immunol 169: $5538-5545,2002$.

21. Dong $\mathrm{H}$ and Chen XM: Immunoregulatory role of B7-H1 in chronicity of inflammatory responses. Cell Mol Immunol 3: 179-187, 2006.

22. Blank C, Brown I, Peterson AC, Spiotto M, Iwai Y, Honjo T and Gajewski TF: PD-L1/B7H-1 inhibits the eVector phase of tumor rejection by $\mathrm{T}$ cell receptor (TCR) transgenic $\mathrm{CD} 8+\mathrm{T}$ cells. Cancer Res 64: 1140-1145, 2004.

23. Sledz CA and Williams BRG: RNA interference in biology and disease. Blood 106: 787-794, 2005.

24. Milhavet O, Gary DS and Mattson MP: RNA interference in biology and medicine. Pharmacol Rev 55: 629-648, 2003. 